\title{
Technique of Kidney Transplantation in Mice with Anti-Reflux Urinary Reconstruction
}

\author{
Paulo N. Martins
}

Department of Surgery, Division of Transplant Surgery, Brigham and Women's Hospital, Harvard Medical School, Boston, MA, USA

\begin{abstract}
Experimental models of organ transplantation play a crucial role in establishing the principles of transplantation immunobiology. Murine transplant models of vascularized organs are particularly useful for immunobiological studies because there are more immunological tools available. However, the technique of kidney transplant in mice is very challenging. A difficult aspect of this model is urinary reconstruction, which is frequently associated to complications. In this article, the technique of mouse kidney transplantation using an anti-reflux system (modified extravesical ureteroneocystostomy) is described and illustrated for the first time. Although technically demanding, this procedure is feasible and may reduce the incidence of urine leakage and reflux.
\end{abstract}

Key words: kidney transplantation; experimental model; mouse; microsurgery

Int Braz J Urol. 2006; 32: 713-20

\section{INTRODUCTION}

Transplant models of vascularized organs in the mouse are particularly useful for immunobiological studies because there are more immunological tools available (inbred, transgenic and knockout animals; genetic mapping, monoclonal antibodies, reagents, etc.) $(1,2)$.

The technique of mouse kidney transplantation was first established by Skoskiewicz \& Russel already in 1973 (3), then improved by Kalina (4) and Zhang (5). However, it is currently performed only in a few transplantation centers in the world, due to the anastomoses complexity (renal artery diameter $0.3 \mathrm{~mm}$ ).

\section{SURGICAL TECHNIQUE}

Male inbred C57BL/6 mice (Jackson Laboratories) weighting 20-30 g were used for kidney transplantations. Animals were housed and cared for in accordance with our institution's guidelines for experimental animals. In a small series of 20 animals, consecutively operated using this technique, the failure rate was $50 \%$ and apparently related to vascular complications.

Mice were anesthetized with a solution of ketamine $(80 \mathrm{mg} / \mathrm{kg}$ i.p.) and xylazine ( $5 \mathrm{mg} / \mathrm{kg}$ i.p.) mixed in a normal saline solution. All procedures were performed at 4-25x magnification using a microscope (SMZ800, Nikon, Japan) and standard microsurgical instruments. 


\section{Donor Operation}

After induction of anesthesia, the mouse was shaved, fixed on the operating board and the abdomen was prepped with betadine. To assure normal volemia, a saline solution was instilled in the abdominal cavity whenever it became dry. A midline incision from the xyphoid appendix to pubis was performed and exposure was achieved using small retractors to keep liver and bowels away from the left kidney.

The renal vein and artery were dissected apart under X20 magnification avoiding direct manipulation of the vessels. Left adrenal and testicular vessels were ligated with 10-0 polypropylene sutures and divided. Then, the kidney was separated from the perinephric fat and adrenal gland, the ureter was dissected freely down to the bladder without stripping its fat and cut with a small (1-2 mm) bladder patch. After clamping the aorta above the renal artery, a 30-gauge needle was introduced into the aorta, the inferior (caudal) vena cava was cut and the graft perfused with $1.0 \mathrm{~mL}$ cold heparinized saline solution $(100 \mathrm{U} / \mathrm{mL})$. Renal vessels were cut with a Carrel patch of the aorta and vena cava and the graft was removed and stored in a normal saline solution at $4^{\circ} \mathrm{C}$ for $20 \mathrm{~min}$. until the time of transplantation (Figure-1).

\section{Recipient Operation}

To increase the success rate, a two-stage procedure was performed, i.e. the left native kidney was removed at the time of grafting and the right native kidney was left in place until day 7 , when the right nephrectomy was performed.

A midline incision from the xyphoid appendix to pubis was performed, and exposure was achieved using small retractors to keep liver and bowels away from the kidney. The renal pedicle was ligated with 7-0 silk suture and the recipient left kidney was removed leaving place for the donor kidney.

After ligating some lumbar branches, the infrarenal aorta and inferior vena cava were isolated and two loops of 7-0 silk were placed proximally and distally around them and tied to promote homeostasis. Elliptical longitudinal aortotomy and cavotomy were performed between these ties and the vessels were flushed to clear blood inside them. Vascular anastomoses were performed end-to-side to the abdominal aorta and vena cava using a running 11-0 nylon suture (Ethilon, Ethicon, USA). To anastomose the renal artery, two stay sutures (11-0 nylon suture) were placed at the artery's opposite ends (inferiorly and superiorly). The back wall was sewn first, through a transluminal approach; then the anterior wall, both with a continuous suture. In general, only four to five stitches on each side are necessary to avoid bleeding.

The vein was anastomosed in the same fashion as the artery. Before suturing the vein, it is important to confirm it is not twisted. Depending on the size of the caval patch, 5 to 7 times the vein was stitched on each side, avoiding pinching the opposite wall. After both anastomoses were performed, the first distal loop was loosened and then the proximal loop. Blood flowed back into the kidney and bleeding was minimal. Saline was usually given subcutaneously proportional to the bleeding (Figure-2).

Urinary reconstruction was established by suturing the small bladder patch to a cystotomy located on the bladder dome (similar to the LichGregoir technique for extravesical ureteroneocystostomy). The animal should be well hydrated to keep the bladder filled. First, the serosa and muscular layers of the posterolateral aspect of the bladder were divided $(5 \mathrm{~mm})$ with the microscissors avoiding major vessels and taking care not to enter the bladder. This develops a plane between the muscularis and mucosa allowing the mucosa to bulge out. Then, the bladder mucosa was divided ( 2 $\mathrm{mm}$ ) and sutured to the mucosa of the patch from the donor's bladder with a running 11-0 nylon suture (Ethilon, Ethicon, USA). Then, one interrupted seromuscular suture was placed in each quadrant of the patch, and finally three interrupted 11-0 nylon seromuscular sutures were loosely placed over the patch to close the bladder muscle over the bladder patch and terminal ureter, burying the anastomosis, sealing the first suture and creating a muscular tunnel to reduce the risk of urine reflux (Figure-3).

Abdominal wall and skin were closed with a continuous 5-0 absorbable suture (Vycryl, Ethicon, USA). A saline $0.5 \mathrm{~mL}$ solution was given subcutaneously after the transplant and no antibiotics 


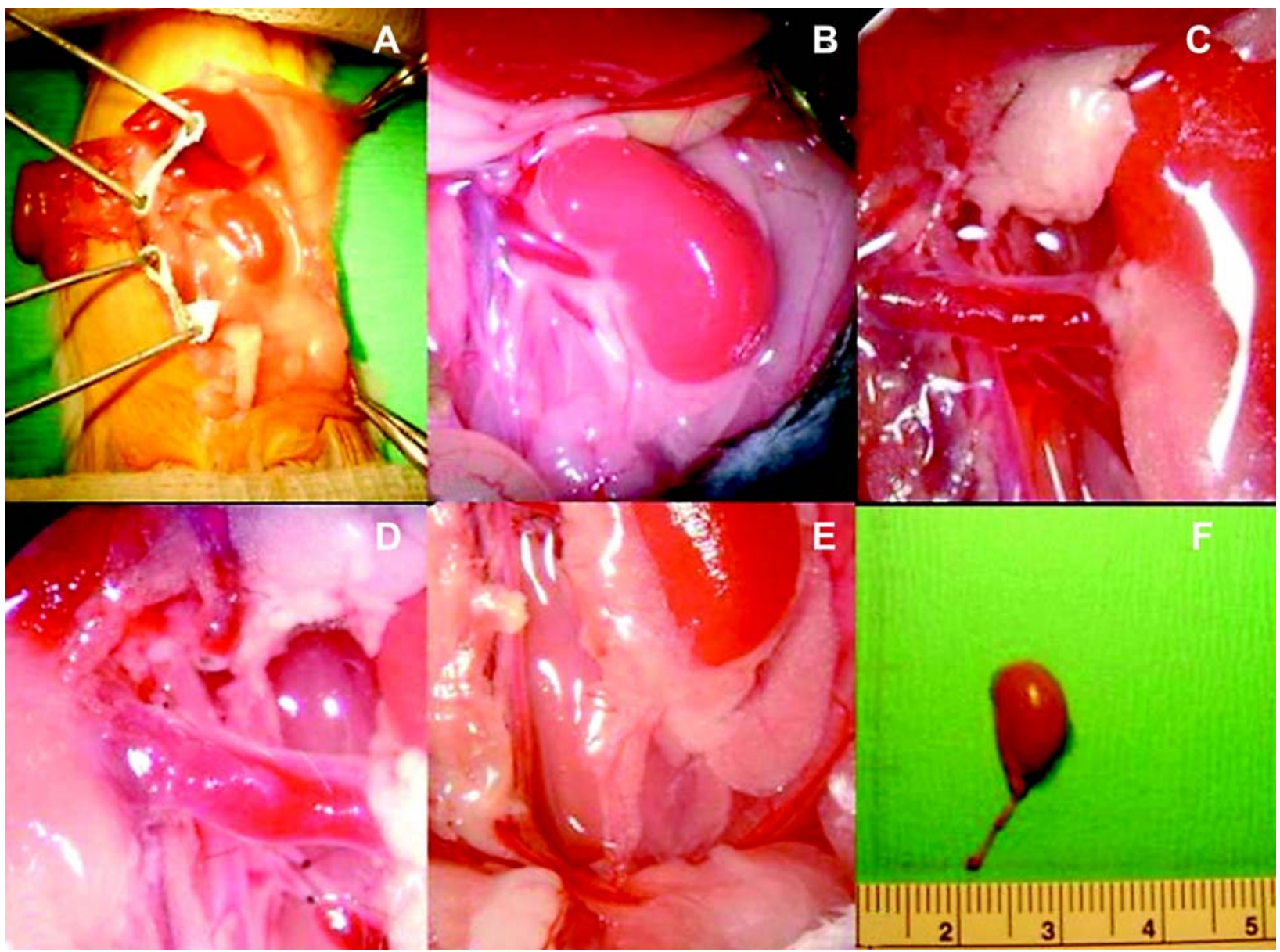

Figure 1 - Donor operation. A and B) Left kidney before dissection $(B, X 4) . C$ ) The renal vein and artery were dissected apart (X8). D) Left adrenal and testicular vessels were ligated and divided (X10). E) The kidney was separated from perinephric fat and adrenal gland, the ureter was dissected freely down to the bladder. After clamping the aorta above the renal artery, a 30-gauge needle was introduced into the aorta, the inferior vena cava was cut and the graft perfused with $1.0 \mathrm{ml}$ cold heparinized saline solution (100U/mL), X4. F) Renal graft shown in relation to a millimetric scale. The renal vessels were cut with a Carrel patch of the aorta and vena cava (not shown) and the ureter removed together with a small $(1-2 \mathrm{~mm})$ bladder patch. Then, the graft was removed and stored in a normal saline solution at $4^{\circ} \mathrm{C}$ while the recipient was prepared $(X 2)$.

or heparin were given. Mice were kept under a heating lamp until they were awake.

\section{COMMENTS}

Mouse kidney transplantation is a very demanding microsurgical procedure full of details, which requires long training and meticulous technique. The murine kidney is extremely sensitive to ischemia/reperfusion injury, and to perform a successful operation the time of anastomosis (warm ischemia) should be less than 35 minutes. Most experiments show that in experienced hands the survival rate is between 40 and $70 \%(3,4)$. The learning curve is slow because the risk of renal artery thrombosis is very high and difficult to avoid, and due to urological complications.

In rat kidney transplantation, continuity of urine flow is reestablished most commonly by end- 


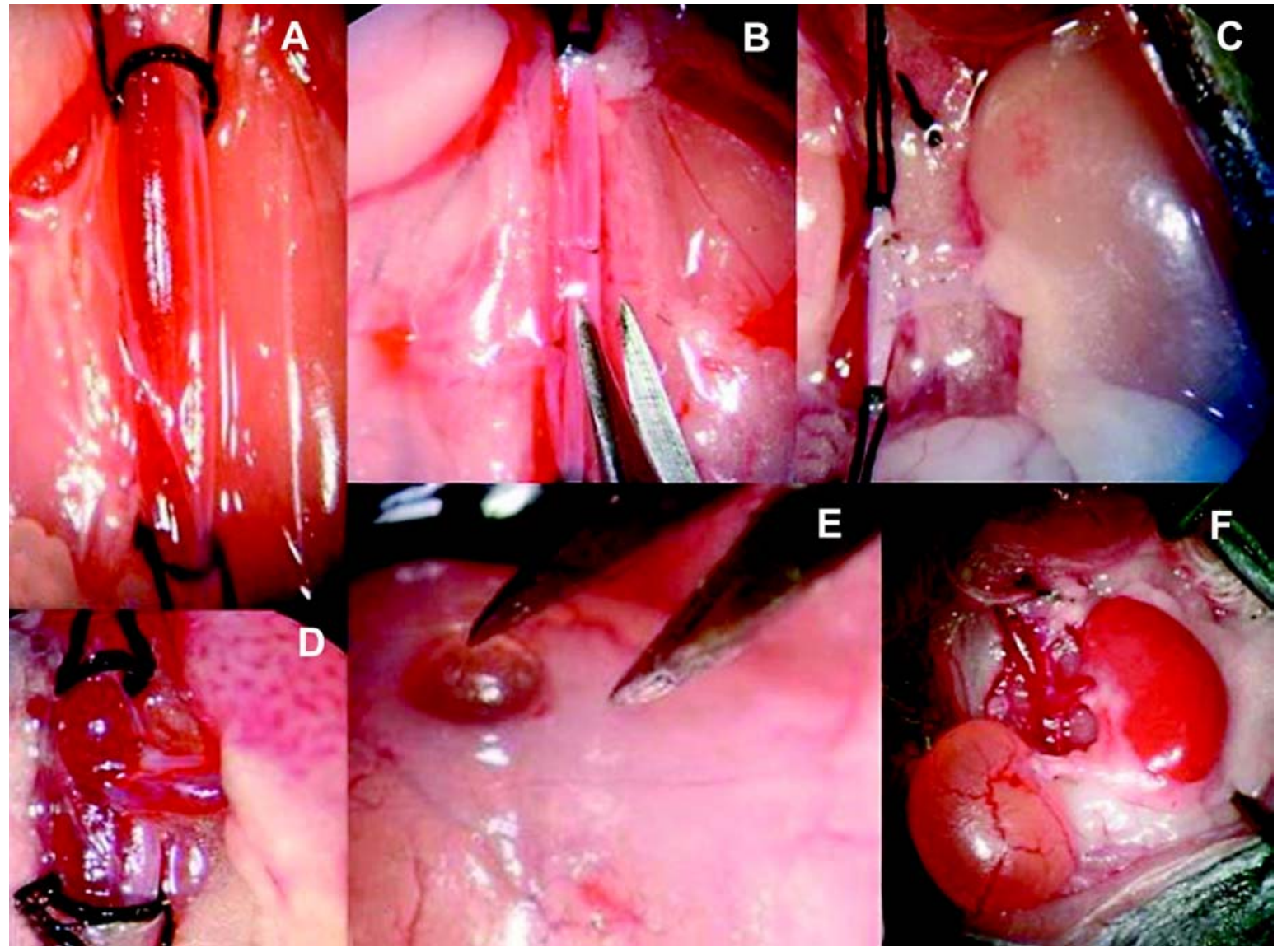

Figure 2 - Technique of mouse kidney transplantation. A) The infrarenal aorta and inferior vena cava were isolated and 2 loops of 7 $O$ silk were placed proximally and distally around them and tied (X12). B) Elliptical longitudinal aortotomy and cavotomy were performed (X10). C) Vascular anastomoses were performed end-to-side to the abdominal aorta and vena cava (X8). D) After both anastomoses were concluded, the loops were loosened and the graft reperfused (X8). E) Urinary reconstruction was established by suturing the bladder patch to a cystotomy located on the bladder dome (X12). F) Kidney graft after anastomosis (X2).

to-end ureter-ureter anastomosis (splinted or nonsplinted). On the other hand, kidney transplantation in mice, probably because of the very small diameter of the ureter, ureter-to-ureter anastomosis has not been described. In the literature, urinary reconstruction in mouse kidney transplantation has been performed either by direct implantation of the ureter in the bladder without anastomosis or most commonly by bladder-to-bladder anastomosis using a large bladder patch. The blood supply of the terminal ureter and bladder patch is exclusive from the graft vasculature and prone to ischemia. Not uncommon, the use of a large bladder patch is associated with either bladder necrosis and urine leakage or neurogenic bladder with calculi formation due to bladder denervation (6).

The most preferred technique for urinary reconstruction in clinical kidney transplantation is extravesical ureteroneocystostomy. Differently from the technique of extravesical ureteroneocystostomy used in the clinic, the author opted for using a small bladder patch since spatulation of the mouse ureter would be difficult. No splint was used since it has been associated with more urological complications (6), and the bladder patch allows enough area to 


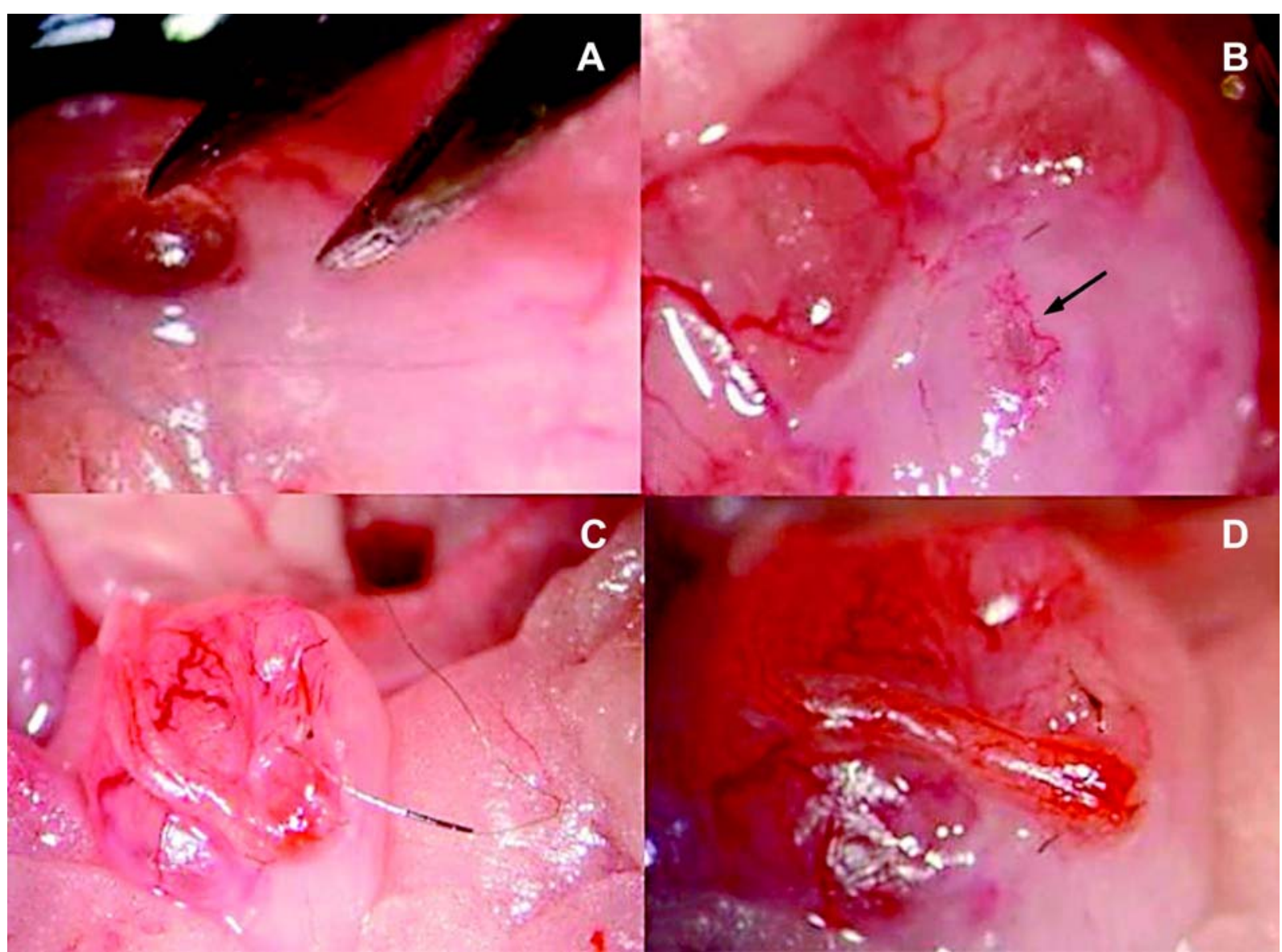

Figures 3 - Urinary reconstruction using a bladder-to-bladder anastomosis and an anti-reflux technique similar to an extravesical ureteroneocystostomy. A) The serosa and muscular layers of the bladder were divided (4 mm) forcing the mucosa to bulge outward (X16). B) and C) A 2 mm incision was performed in the bladder mucosa (arrow) and sutured to the mucosa of the patch from the donor's bladder with a running 11-0 nylon suture (X10 and X8, respectively). D) One interrupted seromuscular suture was placed in each quadrant of the patch, and finally 3 seromuscular stitches were loosely placed to close the bladder muscle over the bladder patch (X10).

place sutures. The technique described here avoids continuous (running) suture of muscular layer that increases ischemia and the risk of patch necrosis and urine leakage. Water-tightness is ensured by minimal vesical opening and continuous suture. The incidence of vesical calculi depends also on the suture material used for urinary reconstruction (6), and therefore, newer absorbable microsutures are preferred.

An anti-reflux system, although timeconsuming and difficult to perform, may reduce the incidence of urosepsis related to urinary fistulae and reduce the risks of hydronephrosis and chronic pyelonephritis in the long-term. To the author's knowledge this is the first report on urinary reconstruction in the mouse using an anti-reflux technique. The potential advantages of this technique applied in the murine model still need to be confirmed in the long-term in case-control studies.

\section{ACKNOWLEDGEMENT}

This work was performed at the CharitéUniversity Medicine Berlin, Virchow Clinic, Department of General Surgery and Transplantation, Berlin, Germany. 


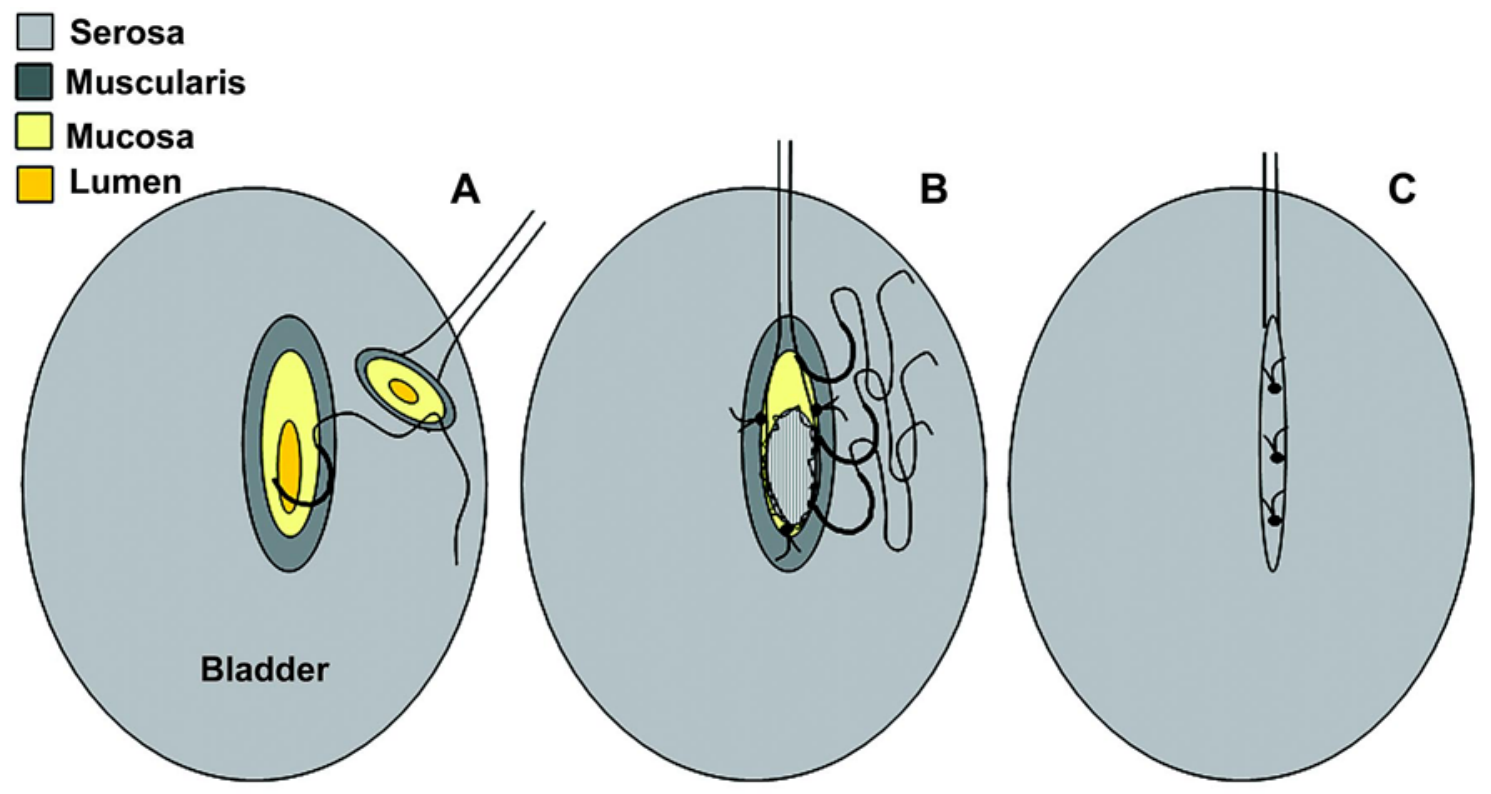

Figure 4-Schematic drawings of the ureterovesical anastomosis steps, as shown in Figure-3.

\section{CONFLICT OF INTEREST}

None declared.

\section{REFERENCES}

1. Martins PN: Importance of microsurgery for organ transplantation. Acta Cir. Bras. 2003; 18: 59-61.

2. Martins PN, Filatenkov A: Microsurgical techniques for experimental kidney transplantation and general guidelines to establish studies about transplantation immunology. Acta Cir Bras. 2003; 18: 355-60.
3. Skoskiewicz M, Chase C, Winn HJ, Russell PS: Kidney transplants between mice of graded immunogenetic diversity. Transplant Proc. 1973: 721-5.

4. Kalina SL, Mottram PL: A microsurgical technique for renal transplantation in mice. Aust N Z J Surg. 1993; 63: 213-6.

5. Zhang Z, Schlachta C, Duff J, Stiller C, Grant D, Zhong $\mathrm{R}$ : Improved techniques for kidney transplantation in mice. Microsurgery. 1995; 16: 103-9.

6. D'Silva M, Gittes RF, Wolf P, Pirenne J, Munger K, Pascual J, et al.: Rat kidney transplantation update with special reference to vesical calculi. Microsurgery. 1990; 11: 169-76.

\section{Correspondence address:}

Dr. Paulo Ney Aguiar Martins

Division of Transplant Surgery

Brigham and Women's Hospital

221 Longwood Ave, Rm 309

Boston, Massachusetts, 02115, USA

Fax: + $1617732-5724$

E-mail:pmartins@partners.org 


\section{EDITORIAL COMMENT}

Clinical kidney transplantation has become the preferred method of renal replacement therapy worldwide. Testing new pharmacological and biological agents, and identifying new methods to diagnose and treat rejection depends on preclinical investigation. The use of large animal models such as the monkey, dog, cat, pig, etc. in transplant research has become problematic due to high cost, the need for specialized animal care, longer gestation and life spans, and cultural sensitivities regarding certain species. Therefore, the rodent is usually preferred. The rat is of sufficient size (300-400 grams) to permit solid organ transplant experiments using microsurgical techniques, but has been less well characterized than the mouse in molecular biology. Since much of the focus on molecular immunology and genetics has been targeted to murine models (monoclonal antibody production, transgenics, knock in and knock out recombinations,

\section{EDITORIAL COMMENT}

The author reports for the first time a modified extravesical ureterocystoneostomy for urinary tract reconstruction in the mouse kidney transplant model. After completion of the vascular anastomosis the bladder dome is incised over a length of $5 \mathrm{~mm}$ avoiding cutting into the mucosa. The mucosa is opened at the distal end of the submucosal tunnel over a distance of approximately $2 \mathrm{~mm}$. The transplant ureter together with its distal end and a small bladder patch is then anastomosed to the mucosa. Thereafter, the muscularis of the bladder wall is readapted over etc.), the mouse is better suited to transplant experimentation. However, the adult mouse (30-50 grams) is $5-10$ times smaller than the rat, more difficult to perform microsurgery, and ultimately more prone to technical failures. A major limiting factor in murine whole organ kidney transplantation has been the ureteral reconstruction. The author offers a nice technique of simplifying the anastomosis of the small ureter by using a donor bladder patch. This technique, similar to the human extravesical ureteral anastomosis, may also limit post transplant urinary infections by creating an antireflux tunnel. Any approach that simplifies microsurgical transplant techniques in murine models would be a welcome addition to this difficult endeavor. This technique should be tried by other murine microsurgical teams to see if diminished technical failures can reinvigorate the wider use of murine kidney transplants for long term models.

\section{Dr. Stuart M. Flechner \\ Director, Clinical Research Section of Renal Transplantation Cleveland Clinic Foundation \\ Cleveland, Ohio, USA \\ E-mail:flechns@ccf.org}

the implanted transplant ureter with 3 interrupted sutures.

Since the first description by Fisher \& Lee, the use of rat-inbred strains as an investigational model of renal transplantation has been extensively used (1). Microsurgical techniques have been developed to the extent that the postoperative survival of renal grafted rats is now routine. However, renal transplantation in mice is technically very demanding due to the small vessels size and the inherent risk of vascular thrombosis and graft loss. Furthermore, 
reconstruction of the urinary tract has been associated with upper urinary tract obstruction as well as ureter and bladder patch necrosis (2). The learning curve in kidney transplantation in mice is substantially longer than in rats and the mortality rate, as stated by the author, is as high as $50 \%$, even in the hands of experienced microsurgeons and long-term survival rates are sparse. A survival rate of more than 4 weeks has been reported by the same author in only 4 animals $(8 \%)$ in a consecutive series of 50 kidney transplant procedures in mice using a bladder to bladder patch for urinary tract reconstruction (3). Most animals died due to surgical related complications.

Therefore, it seems of utmost importance to balance the advantages and disadvantages of performing a technical demanding antireflux procedure during kidney transplantation in mice. Prolonged surgical time in small animals, as for performing an extravesical ureterocystoneostomy, may lead to hypothermia and eventually death and has to be considered as a major risk factor (4). Further, performing an antireflux procedure does not inevitably allow drawing the conclusion that there is no vesicoureteral reflux and that the graft is protected against the possibility of upper urinary tract infection. In humans the key to success to prevent vesicoureteral reflux according to Grégoir is to create a submucosal tunnel of 5 to $6 \mathrm{~cm}$ length (5). However, it remains to be determined how long the submucosal tunnel has to be in mice to prevent vesicoureteral reflux.

\section{REFERENCES}

1. Fisher B, Lee S: Microvascular surgical techniques in research, with special references to renal transplantation in the rat. Surgery. 1965; 58: 904-14.

2. Russell PS, Chase CM, Colvin RB, Plate MD: Kidney transplantation in mice: an analysis of the immune status of mice bearing long-term, H-2 incompatible transplants. J Exp Med. 1978; 22: 1449-68.

3. Martins PN: Learning curve, surgical results and operative complications for kidney transplantation in mice. Microsurgery. 2006 (ahead of print).

4. Pahlavan PS, Smallegange C, Adams MA, Schumacher M: Kidney transplantation in rats: assessments, complications and management. Microsurgery. 2006; 26 : 404-11.

5. Grégoir W: The surgical treatment of congenital vesicoureteral reflux. Acta Chir Belg. 1964; 63: 431-9.

Dr. Martin Schumacher FMH Urologie, FEBU Urologkliniken

Karolinska University Hospital SE-171 76 Stockholm, Sweden E-mail: Martin.Schumacher@insel.ch 